\title{
Size distribution of black spot defects and their contribution to swelling in irradiated $\mathrm{SiC}$
}

\author{
B. Tyburska-Püschel ${ }^{1, a}$, Y. Zhai ${ }^{2}$, L. $\mathrm{He}^{2}$, C. Liu ${ }^{2}$, \\ A. Boulle ${ }^{3}$, P.M. Voyles ${ }^{2}$, I. Szlufarska ${ }^{1,2}$, K. Sridharan ${ }^{1}$

Keywords: 4H-SiC, swelling, black spot defects, XRD, TEM, strain, ion-irradiation, carbon, krypton PACS: $81.05 \mathrm{Je}, 81.40 \mathrm{Jj}, 83.85 \mathrm{Hf}$

\footnotetext{
${ }^{a}$ Corresponding author: B. Tyburska-Püschel, tyburska (at) engr.wisc.edu, tel.: +1-608-890-3578 


\section{INTRODUCTION}

Excellent mechanical and thermal properties as well as outstanding radiation resistance have secured silicon carbide ( $\mathrm{SiC}$ ) numerous applications especially in demanding areas like nuclear systems (TRISO, fusion blanket), space telescopes, and airplane engines [1]. In the case of light

30 water reactors (LWRs), SiC is envisioned as an accident-tolerant cladding capable of withstanding high radiation doses and pressures. Such harsh conditions will expose the material to high strains and stresses leading to swelling and creep. The swelling of $\mathrm{SiC}$ at various temperatures and damage levels has been studied extensively [2] and is fairly well understood quantitatively. However, in order to develop predictive tools for swelling modeling under various reactor conditions, the underlying mechanisms have to be studied especially at LWRs operation temperatures, below $1000^{\circ} \mathrm{C}$, where swelling is dominated by point defect formation and clustering. Previous results on neutron and silicon-irradiated $\mathrm{SiC}$ at medium-to-high damage levels (4.4 - $100 \mathrm{dpa})$ and temperatures of $600-1400{ }^{\circ} \mathrm{C}[3,4]$ have shown that qualitative study of swelling via conventional transmission electron microscopy (TEM) is insufficient, as the observed defects account only for about $10 \%$ of estimated total value [4]. It was therefore suggested that so-called black spot defects (BSDs - small interstitial clusters), observed by some researchers as patches of dark TEM contrast, contribute to the remainder of the swelling [2].

In this work, we improve on the qualitative description of swelling by accounting for black spot defects and the rigid substrate effect. High-resolution TEM was employed to study BSD size and density in ion-irradiated $4 \mathrm{H}-\mathrm{SiC}$. Based on the TEM data we calculate contributions to swelling from the observed black spot defects and from point defects that are expected to be present at these irradiation conditions based on previous modeling studies. We compare these results to swelling estimated by X-ray diffraction (XRD) experiments. The swelling calculation from XRD is corrected by accounting for the effect of the un-irradiated substrate on the lateral confinement of the damage zone.

\section{EXPERIMENTAL APPROACH AND MODELING DETAILS}

\section{A. Material}

$\{0001\}$ single crystal, hexagonal 4H-SiC $(a=3.073 \AA, c=10.053 \AA)$, n-doped, $4.1^{\circ}$ off towards $[11 \overline{2} 0] \pm 0.5^{\circ}$, with low micropipe and double-side polish from Cree was chosen to study black 
spot defects. Single-crystal was selected for easy strain and swelling measurements by XRD and to reduce the defect density in the virgin material for TEM investigations.

\section{B. Ion-irradiation}

To simulate neutron irradiation, $4 \mathrm{H}-\mathrm{SiC}$ was irradiated at $600{ }^{\circ} \mathrm{C}, 800{ }^{\circ} \mathrm{C}$, and $950{ }^{\circ} \mathrm{C}$ by 3.15 $\mathrm{MeV} \mathrm{C}^{2+}$ up to $0.4 \mathrm{dpa}$ and $1 \mathrm{MeV} \mathrm{Kr}^{+}$at $600^{\circ} \mathrm{C}$ and $800^{\circ} \mathrm{C}$ up to 0.4 and $0.8 \mathrm{dpa}$. Irradiations were performed at conditions at which BSDs are expected to form without introducing much strain [2].

Carbon irradiation was performed using the 1.7 MV tandem accelerator located at UW-Madison under the following conditions: $3.15 \mathrm{MeV} \mathrm{C}^{2+}$ (projected range $R_{\mathrm{p}}=2.23 \pm 0.11 \mu \mathrm{m}$ ) up to a fluence of $5.14 \times 10^{16} \mathrm{C} / \mathrm{cm}^{2}$ which corresponds to $0.4 \mathrm{dpa}$ at the depth of $1 \mu \mathrm{m}$ (see Fig. 1), assuming threshold displacement energies of $20 \mathrm{eV}$ and $35 \mathrm{eV}$ for $\mathrm{C}$ and $\mathrm{Si}$ [5], respectively. For the damage level calculation, the method proposed by Stoller et al. [6] was employed. The maximum C concentration is about 3 at.\% with a majority of the $\mathrm{C}$ interstitials located at the end of the irradiation range. The average current was around $4 \mu \mathrm{A}$, and the flux was kept at a level of $6.5 \times 10^{12} \mathrm{C} /\left(\mathrm{cm}^{2} \mathrm{~s}\right)$, resulting in a damage rate of $5 \times 10^{-5} \mathrm{dpa} / \mathrm{s}$. The beam was rastered $(64 \mathrm{~Hz}$ horizontally, $517 \mathrm{~Hz}$ vertically) over the entire irradiation area, and its uniformity was controlled by an infrared camera. Sample temperature, controlled by two thermocouples attached diagonally to the sample holder, was attained by external and beam heating with fluctuations of $\pm 20 \mathrm{~K}$. The background pressure during irradiation was kept around $10^{-6}$ Torr.

$1 \mathrm{MeV} \mathrm{Kr}$-irradiations $\left(R_{\mathrm{p}}=0.4 \pm 0.09 \mu \mathrm{m}\right)$ were performed at the University of Illinois, Urbana-Champaign, Frederick Seitz Material Research Laboratory using an HVEE van der Graaf accelerator. The irradiations were conducted up to a fluence of either $3 \times 10^{14} \mathrm{Kr} / \mathrm{cm}^{2}$ or $6 \times 10^{14} \mathrm{Kr} / \mathrm{cm}^{2}$, which corresponds to 0.4 and $0.8 \mathrm{dpa}$ at the damage peak, respectively (see Fig. 1). The maximum $\mathrm{Kr}$ concentration was about 0.03 at. $\%$ (for $6 \times 10^{14} \mathrm{Kr} / \mathrm{cm}^{2}$ ), which does not alter the stoichiometry of the implanted SiC samples. The implantation spot was $6 \times 6 \mathrm{~mm}^{2}$, and the flux varied between $1.4 \times 10^{12} \mathrm{Kr} /\left(\mathrm{cm}^{2} \mathrm{~s}\right)$ and $3 \times 10^{12} \mathrm{Kr} /\left(\mathrm{cm}^{2} \mathrm{~s}\right)($ current $80-170 \mathrm{nA})$ yielding a damage rate of $1.7 \times 10^{-3} \mathrm{dpa} / \mathrm{s}$ to $4 \times 10^{-3} \mathrm{dpa} / \mathrm{s}$. The background pressure was around $5 \times 10^{-7}$ Torr, and the sample temperature was measured by a K-type thermocouple attached to the sample holder. The temperature uncertainty was within $\pm 5 \mathrm{~K}$. The same method of damage level calculation was employed as for C-irradiation. During the irradiation, all samples were attached 
to the sample stage using silver paint, but only one sample was irradiated at a time while the other three were kept heated during this period. Once the desired fluence was reached, the beam was shifted to the next sample. In all cases, the beam was perpendicular to the sample surface, and ion channeling was avoided as a consequence of the $4.1^{\circ}$ off-cut angle of the virgin material.

\section{XRD measurements}

Ion-induced strain and point defect swelling were measured by means of XRD. The measurements of single crystal $4 \mathrm{H}-\mathrm{SiC}$ were conducted at room temperature with a PANalytical X'pert PRO diffractometer $(45 \mathrm{kV}, 40 \mathrm{~mA})$ in Bragg (reflection) geometry utilizing $\mathrm{Cu}_{\mathrm{K}_{\alpha 1}}$ radiation $(\lambda=0.154056 \mathrm{~nm})$ in combination with a hybrid monochromator consisting of a closely coupled X-ray mirror and a 4-bounce Ge 220 monochromator (18 arcsec resolution). By adjusting azimuthal and polar angles, the sample was precisely oriented in order to align a given crystallographic direction exactly with the diffraction vector. The alignment was performed on the crystal planes instead of the surface, which reduced the axial divergence. In the case of the off-cut samples used in this research, the XRD curves were measured at an angle $\alpha=4.1^{\circ}$ from the reciprocal lattice vector corresponding to the (0004) pole with the [1120] direction (off-cut angle direction) in the detection plane. The $2 \theta-\omega$ scans were taken at the $(0004)$ pole with $0.001^{\circ}$ steps, $0.5 \mathrm{~s}$ per step. To determine whether there is any in-plane compressive stress, reciprocal space maps (RSMs) were recorded in the vicinity of the (004) and (106) reflection.

The value of the total normal strain was calculated from the equation

$$
\varepsilon_{\mathrm{N}}^{\mathrm{tot}}=\frac{\Delta c}{c}=-\Delta \theta \cot \theta_{\mathrm{B}}
$$

105 $c=10.053 \AA$.

To determine the fraction of the damage zone being probed by X-rays, we calculate the sample thickness $x$ required to reduce the amount of transmitted X-ray intensity by half (the so-called half-value layer). The mass absorption coefficients for X-ray $\mathrm{Cu}$ cathode radiation in $\mathrm{C}$ and $\mathrm{Si}$ are $4.51 \mathrm{~cm}^{2} / \mathrm{g}$ and $63.7 \mathrm{~cm}^{2} / \mathrm{g}$, respectively, giving $45.94 \mathrm{~cm}^{2} / \mathrm{g}$ for $\mathrm{SiC}$ [7]. These values give the linear absorption coefficient $\mu_{\mathrm{SiC}}=147.5\left[\mathrm{~cm}^{-1}\right]$ and $x \approx 15 \mu \mathrm{m}$ at $\omega=15^{\circ}$, and the X-rays thus penetrate both the substrate and the damage zone. 


\section{TEM procedure}

\section{E. Method for examination of BSDs}

BSDs appear as nanoscale black features in bright-field TEM images. Identifying BSDs using a global intensity threshold for the entire image has limited accuracy due to background contrast variability, presumably from strain, in the ion-irradiated sample. Therefore, we adopt a method that identifies BSDs using local intensity thresholds, which compares BSDs only to their immediate environment. The method was implemented in MATLAB and used for the analysis of a few hundred BSDs per irradiation condition.

Figure 2(a) shows a gray-scale bright-field TEM image. Visually, we identify the black features as BSDs, but they are surrounded by gray areas which we do not include in the BSDs. The first step is to find all the possible strained, BSD-containing areas, which we do with a relatively high intensity threshold value (128 in this case). Figure 2(b) shows the thresholded binary image of (a), with all the pixels with gray values smaller than 128 shown in black, and other areas shown in white. All BSDs are captured, but so is a significant area of strained material outside the BSDs.

In a second step, we use conventional particle analysis techniques to segment the image in Figure 2(b) and then apply a second, local threshold equal to the mean gray value inside each identified region. Figures 2(c) and (d) show the subregions inside each region from (a) and (b) that also lie below the local threshold. In Fig. 2(c), the regions are marked in green. Figure 2(d) is the corresponding binary image. All of the BSDs are identified, and isolated BSDs are near the 
right size. However, nearby pairs or larger groupings of BSDs are merged because the intensities of a few pixels at their boundaries are always lower than the pixels around their centers. In a third step, we repeat the local average intensity threshold to separate these merged defects, resulting in the final identified BSDs shown in Figures 2(e) and 2(f). Figure 2(f) is then segmented using the same particle analysis techniques to determine the area of each BSD, which is converted into an approximate diameter for the equivalent circle.

\section{F. Modeling swelling based on TEM data}

Swelling is calculated based on the BSD diameter $d$ and number density $C_{\mathrm{BSD}}$ distribution determined by TEM (see Fig. 7). For simplicity, we assume that each interstitial cluster is spherical and stoichiometric. The number density $C_{\mathrm{CTI}}$ of cluster-trapped interstitials in BSDs is calculated by dividing the total cluster volume by the average $\Omega$ of the $\mathrm{C}$ and $\mathrm{Si}$ atomic volumes in $\mathrm{SiC}$. There are no vacancy clusters observed in our samples, which is consistent with previous studies showing that void swelling starts at $1100{ }^{\circ} \mathrm{C}-1250^{\circ} \mathrm{C}$ [3]. Contributions to swelling come also from 6 types of isolated point defects (IPD) which are present in the sample but not visible in TEM. These isolated point defects are: interstitials $\left(\mathrm{C}_{\mathrm{I}}\right.$ and $\left.\mathrm{Si}_{\mathrm{I}}\right)$, vacancies $\left(\mathrm{V}_{\mathrm{C}}\right.$ and $\left.\mathrm{V}_{\mathrm{Si}}\right)$, and antisite defects $\left(\mathrm{C}_{\mathrm{Si}}\right.$ and $\left.\mathrm{Si}_{\mathrm{C}}\right)$. The concentrations of these defects are determined based on our cluster dynamics model reported in Ref. [8]. Cluster dynamics model was developed to reproduce size distribution of clusters with diameters smaller than $2.5 \mathrm{~nm}$, which were dominant in the experimental samples from [8]. Ratios between the numbers of isolated point defects and cluster-trapped interstitials $R$ are calculated and reported in Table I. Using the same cluster dynamics model, it was found that for the different experimental conditions considered in Ref. [8] these ratios do not vary by more than a few percent. For consistency we use the same set of ratios (shown in Table I) for all swelling calculations. Total swelling $S_{\text {total }}$ is thus estimated by adding formation volumes $V_{\text {IPD }}$ of all the defects present in the system (including clusters and isolated point defects) using the following equation:

$$
S_{\text {total }}=S_{\mathrm{BSD}}+S_{\mathrm{IPD}}=C_{\mathrm{CTI}} \Omega+\sum_{i=1}^{6} C_{\mathrm{CTI}} R^{i} V_{\mathrm{IPD}}^{i}=\frac{\pi}{6} \sum_{n} d_{n}^{3} \frac{C_{\mathrm{BSD}}}{n} \frac{\Omega+\sum_{1}^{6} R^{i} V_{\mathrm{IPD}}^{i}}{\Omega}
$$

where $n$ is the number of detected BSDs per sample, an experimental value which is different for each sample, $i$ denotes a type of defect, and $\Omega=10.008 \AA^{3}$. Formation volumes of isolated point defect have been calculated in Ref. [9] and are also quoted in Table I. 


\section{RESULTS}

\section{A. Swelling results by XRD}

Figure 3 shows $2 \theta-\omega$ scans from C-irradiated $4 \mathrm{H}-\mathrm{SiC}$ plotted as a function of the scattering angle (the bottom horizontal axis) and the total elastic strain normal to the surface (the top hori-

175 zontal axis). An XRD curve from an un-irradiated sample is shown as a reference. The main sharp Bragg peak of (0004) and intensity - denoted as $I_{0}$ - is located at $2 \theta=35.7^{\circ}$ and comes from the unperturbed SiC crystal. All XRD curves exhibit the substrate peak. However, for the irradiated samples this peak becomes broader, which can be especially visible at the lowest temperature of $600^{\circ} \mathrm{C}$. For Kr-irradiation (see Fig. 4) the substrate peak is broadened as well, however, to a lesser extent than for the $\mathrm{C}$ case. The broadening of the substrate peak is large but not unusual. Examining the $2 \theta-\omega$ scans published by other researchers [10-13] one can observe some broadening of the substrate peak is rather common and occurs even when the ratio of the damage zone thickness to the X-ray penetration depth is large. Our peak broadening, as explained in the XRD modeling section, stems from random lattice spacing fluctuations. RSMs around (004) and (106) (not shown) indicate lack of compressive stress as the substrate and the satellite signal have the same value of the in-plane $Q_{\mathrm{x}}$ component of scattering vector. Both peaks are broaden (along $Q_{\mathrm{x}}$ ), which indicates a presence of highly defective microstructure with the defects being identify as BSDs (by TEM) and point defects (cluster dynamics) (See Sec. III D, IV B).

After irradiation, a new, asymmetric, broad peak on the low-angle side appears (see Figs. 3 and $4 \mathrm{a}$ ), coming from the damaged part of the crystal. Within the damage zone, irradiation causes a loss of the long-range order due to accrual of point and black spot defects, which gives rise to elastic strain and swelling. The location of the satellite peak (see Figs. 3 and 4a) provides information on irradiation-induced strain. Position of the satellite peak at $2 \theta<2 \theta_{\mathrm{B}}$ indicates an increase of the interplanar distance, which means that the implanted layer undergoes tensile strain along the direction normal to the surface of the crystal and along the irradiation direction. The lack of fringes is caused by the curve broadening due to root-mean-squared (rms) strains. The width of the damage peak is inversely proportional to the width of the damaged zone with the given level strain. Since the satellite peak for $\mathrm{C}$ irradiation is broader than for $\mathrm{Kr}$, this indicates that the region with the maximum strain is narrower. As shown in Fig. 3, strain builds up with decreasing temperature, which indicates the presence of simple defects (e.g., interstitials and vacancies) and a 
lack of extended defects, which can induce plastic relaxation. As also seen in Fig. 3, the competitive effect of defect creation and annihilation shifts the satellite peak progressively towards higher $2 \theta$ angles with increasing irradiation temperature. However, even a temperature of $950^{\circ} \mathrm{C}$ is not sufficient to ensure complete defect recovery.

\section{B. Total normal strain vs. true normal strain}

In the case of the Kr-irradiated $4 \mathrm{H}-\mathrm{SiC}$ (see Fig. 4a), estimating the maximum strain is straightforward, as the XRD peaks are fairly sharp, and the intensity of the tail signal from the satellite peak (the region above the tensile strain value of $0.9 \%$ ) is comparable to the tail intensity for the undamaged sample. The case of $\mathrm{C}$-implanted $\mathrm{SiC}$ is more complicated, because the satellite peaks are much broader, and the intensities of the tails of the low-angle satellite peaks are higher than for the undamaged sample, even at strain values above $1.5 \%$ (not shown). To estimate the maximum strain, a Gaussian function was fitted to the satellite peaks, and the expected value defined the total in-plane strain $\varepsilon_{\mathrm{N}}^{\text {tot }}$, with the standard deviation as strain error. The obtained $\varepsilon_{\mathrm{N}}^{\text {tot }}$ is a measure of the elastic strain produced in a thin layer laterally confined by the unperturbed substrate. This total strain differs from the elastic strain that would be measured in a freestanding solid (e.g., as for TEM samples and as in the case of neutron-irradiated experiments) and therefore yields only approximate values of swelling [10, 14-16]. To find the true strain in the damaged zone $\varepsilon_{\mathrm{N}}^{\mathrm{d}}$ and separate it from the "substrate strain" $\varepsilon_{\mathrm{N}}^{\mathrm{s}}$ (result of Poisson expansion), we follow the formalism proposed by Debelle and Declémy [17] adjusted to hexagonal $\langle 0001\rangle$-oriented system, in which the true and the total normal strains are related through the elastic constants as follows

$$
\begin{aligned}
\varepsilon_{\mathrm{N}}^{\mathrm{tot}} & =\varepsilon_{\mathrm{N}}^{\mathrm{d}}+\varepsilon_{\mathrm{N}}^{\mathrm{s}}, \\
\frac{\varepsilon_{\mathrm{N}}^{\mathrm{d}}}{\varepsilon_{\mathrm{N}}^{\mathrm{tot}}} & =\frac{C_{11}+C_{12}-C_{13}}{C_{11}+C_{12}+C_{13}} .
\end{aligned}
$$

Using the elastic constants $C_{11}, C_{12}$, and $C_{13}$ for $4 \mathrm{H}-\mathrm{SiC}$ from Kamitani et al. [18], we estimate that the true strain is $84 \%$ of the total normal strain (see Tab. II). Both for C- and Kr-irradiation, the strain values decrease with increasing irradiation temperature, indicating defect annealing. At $600{ }^{\circ} \mathrm{C}$, the strain measured in the $\mathrm{Kr}$-irradiated material is about $27 \%$ higher than in the $\mathrm{C}$ irradiated samples. However, at $800^{\circ} \mathrm{C}$ the strain values for both types of ions are comparable. $\mathrm{Kr}$-irradiation above 0.4 dpa at either temperature does not result in an increase of the strain value, 
suggesting defect saturation.

\section{XRD data modeling}

Ion-irradiation entails variation of the spacing of the lattice planes as well as atom displacements from their lattice sites. The first $\left(\varepsilon_{\mathrm{N}}^{\text {tot }}\right)$ results in an angular shift of diffracted intensity from that of the unperturbed substrate, while the second (Debye-Waller factor) lowers the structure factor of the damaged region. We attempt to reproduce the strain profile by modeling the spectra of $1 \mathrm{MeV}$ Kr-irradiated $4 \mathrm{H}-\mathrm{SiC}$ at $600{ }^{\circ} \mathrm{C}$ up to $0.8 \mathrm{dpa}$ (see Fig. $4 \mathrm{~b}$ ) using least-square fitting based on the approach proposed by Boulle and Debelle [19].

The lack of specific features, such as interference fringes, inhibits the possibility of an absolute determination of strain profile. Therefore, SRIM's damage profile was used as an initial condition for the strain profile modeling and its shape was refined until agreement with the experimental data was reached. Fitting was done using a classical least-square algorithm, which is a local search around the starting point. Constraints were placed on the maximum possible value of the strain based on the position of the satellite peak. The results show that the irradiated and the underlying pristine crystal are heterogeneously strained. Hence, in addition to the strain profile obtained (see Fig. 4b), there also are some random lattice spacing fluctuations, which turn out to be normally distributed with a root-mean-squared strain of approximately $0.056 \%$, which account for the broadening of both the substrate and the damage peak. The strain profile in the damaged sample is almost flat $\left(\varepsilon_{\mathrm{N}}^{\text {tot }} \approx 0.9 \%\right)$, which suggests that the sample was implanted above the defect saturation damage level. In Fig. 4b, a narrow region in slight compression (i.e., with negative strain) is noticeable close to the surface $(<15 \mathrm{~nm})$. This region accounts for the high intensity at the right-hand side of the un-irradiated Bragg peak. Excluding this region, the fitted data results in a purely tensile strain profile. However, since the measured data is heavily broadened, an absolute determination of the strain profile is not possible. 


\section{TEM results}

Figure 5 shows bright-field TEM images of $4 \mathrm{H}-\mathrm{SiC}$ irradiated at $800{ }^{\circ} \mathrm{C}$ to (a) $0.51 \mathrm{dpa}$ with $\mathrm{C}$ ions and (b) $0.21 \mathrm{dpa}$ with $\mathrm{Kr}$ ions. BSDs have been studied with TEM for decades, but their structure is still not clear. For instance, Price [20] found defects on $\{111\}$ planes similar to BSDs and identified them as Frank dislocation loops; Senor et al. [21] and Katoh et al. [3] reported these undetermined black dots as a mix of small dislocation loops. As shown in Figure 5, we detect BSDs as mostly circular or slightly oval dark-contrast strains. This shape indicates that there is limited overlap in BSDs through the sample thickness. Because overlap is random, partial overlap would create globular, high curvature defects, as shown by Wang et al. [22]. Estimating the uncertainty in defect concentration created by defect overlap is difficult, but we expect the uncertainty to be dominated by counting statistics. Figure 6 summarizes the average BSD size and number density both from our work and from literature data [3, 20, 21, 23, 24] as a function of irradiation temperature. The label next to each data point indicates its damage level in dpa. Since different researchers use various methods for damage level calculations, we recalculated their values according to our procedure stated in Sec. II B to provide a unified method of damage level calculation. For short range ion-irradiation (Kr-this work and [15]; He-[10, 14, 16]), the damage level is given at the peak value, and for long-range (C-this work, $\mathrm{Si}-[24])$ at a depth of $1 \mu \mathrm{m}$. For neutron-irradiation, we follow Ref. [3] and assume that a fluence of $1.0 \times 10^{21} \mathrm{n} / \mathrm{cm}^{2}$ results in $1 \mathrm{dpa}$. The mean diameters of BSDs in our ion-irradiated samples are around $1.5 \mathrm{~nm}$, and the BSD number densities are on the order of $10^{23} \mathrm{~m}^{-3}$. Sizes are assigned a uniform uncertainty of $\pm 0.1 \mathrm{~nm}$ based on the uncertainty of edge-finding in the image analysis procedure employed. Number density uncertainties are the results of uncertainties of the number counting divided by the volume of the investigated sample section. For Kr ions (see Fig. 6), one can observe the defect diameter to increase modestly but consistently as a function of increasing temperature, while the number density either remains unchanged (for $0.4 \mathrm{dpa}$ ) or increases as well (for $0.8 \mathrm{dpa}$ ). On the other hand, with increasing temperature, the average BSD size in C-irradiated samples remains the same, while their average number density decreases more steeply.

Figure 7 shows the BSD size distribution in all $\mathrm{Kr}$ - and $\mathrm{C}$-irradiated $4 \mathrm{H}-\mathrm{SiC}$ samples. For both $\mathrm{C}$ - and $\mathrm{Kr}$-irradiated samples at any temperature, the highest density of BSDs corresponds to BSDs with a diameter between $0.5-1 \mathrm{~nm}$. For C-irradiation, the BSD number density at a given diameter is always the highest for $600{ }^{\circ} \mathrm{C}$ and is decreasing with increasing temperature. For $\mathrm{Kr}$ 
this trend is followed only at $0.4 \mathrm{dpa}$ at BSD sizes smaller than $1 \mathrm{~nm}$. Otherwise, the BSD number density at a given size is higher at $800^{\circ} \mathrm{C}$ than at $600{ }^{\circ} \mathrm{C}$ for both 0.4 and $0.8 \mathrm{dpa}$. Comparing $\mathrm{C}$ and $\mathrm{Kr}$-irradiation at $0.4 \mathrm{dpa}$ at either $600{ }^{\circ} \mathrm{C}$ or $800^{\circ} \mathrm{C}$, one can observe that $\mathrm{C}$-irradiation results in a higher BSD number density than Kr-irradiation at the same BSD size. For Kr-irradiation, the BSD number density at the same BSD diameter is almost always higher for 0.8 dpa than for 0.4 dpa for both irradiation temperatures.

\section{DISCUSSION OF SWELLING IN SIC}

\section{A. Particle effect}

$\mathrm{XRD}$ results show that for both $\mathrm{C}$ - and $\mathrm{Kr}$-irradiation the increase of irradiation temperature leads to a reduction of tensile strain. In the case of $\mathrm{C}$-irradiation this is achieved through a drop in BSD number density with rising temperature (see Fig. 7) rather than through a BSD diameter decline-the latter stays almost constant over the studied temperature range (see Fig. 6). For Krirradiation, the decrease of strain with temperature cannot be explained by the TEM data - the average BSD size increases at damage levels of both 0.4 and 0.8 dpa (see Fig. 6), and the BSD number density also increases with temperature for almost all sizes of BSD (see Fig. 7) at constant dpa. Additionally, XRD and TEM show discrepancies in terms of the damage level effect studied in Kr-irradiated samples. A higher damage level (at both $600^{\circ} \mathrm{C}$ and $800{ }^{\circ} \mathrm{C}$ ) causes no shift of the satellite peak, which indicates no change in strain and implies defect saturation. However, TEM clearly shows an increase in BSD number density with higher dpa (see Fig. 7) at both temperatures and small growth of the average BSD size (see Fig. 6). The reason for this discrepancy is that TEM is a local examination method, and its results cannot be expected to be fully representative of the entire damaged region probed by XRD. The solution, though time consuming and expensive, would be to examine the damaged zone region at various depths from the irradiated surface to see how BSD size and concentration vary along the damage profile.

At $600{ }^{\circ} \mathrm{C}$ and $0.4 \mathrm{dpa}$, the measured XRD strain is lower in C-irradiated samples. In contrast, at $800{ }^{\circ} \mathrm{C}$ and $0.4 \mathrm{dpa}$, it is the same as for $\mathrm{Kr}$-irradiation (see Tab. II), despite the fact that the average BSD size (see Fig. 6) and concentration at all BSD diameters (see Fig. 7) at a given temperature are higher than for $\mathrm{Kr}$. This observation can be explained by the fact that $\mathrm{C}$ is a self-ion and as such 
can form $\mathrm{C}_{\mathrm{Si}}$ anti-site defects, which have a negative formation volume (they decrease strain, see

Tab. I). Leclerc et al. [25] observed that that strain-to-dpa ratio decreases with the projected range. However, this observation is valid only if the shape of strain profile is comparable the damage profile predicated by SRIM and therefore might not pertain to our results were defect diffusion (which leads to formation of BSDs) and recombination takes place as it is expected at such high temperatures. To clarify the influence of particle type on strain evolution, the particle energies have to be chosen in a way which results in similar SRIM profiles.

\section{B. Difference between XRD and TEM}

The values of swelling from XRD and TEM are shown in Table II and are compared to the previously published data in Fig. 8. The label next to each data point indicates its damage level as calculated according to the procedure mentioned in Sec. IIID. It is evident that the magnitude of swelling decreases with increasing irradiation temperature up to $1100{ }^{\circ} \mathrm{C}$, which is a sign of small defect recovery and lack of defect growth due to its migration. No definite conclusion can be made about the effect of damage level or damage rate (type of irradiation particles) on the magnitude of swelling. However, in general it seems that swelling values derived from the total in-plane strain are higher than from other methods like TEM or linear dimension change, which is mainly due to the fact that the effect of substrate is unaccounted for in the XRD method. Our swelling values as calculated from XRD and TEM are on the same order of magnitude, however in most cases TEM data underestimated XRD predictions by $40 \%$ to $70 \%$, which is also connected to the fact that in TEM lamellae some stresses are relieved.

The likely reason for this discrepancy is that there are probably smaller clusters other than BSD present in the irradiated material, which may be invisible to TEM. Such clusters have been reported based on observations from high-resolution scanning transmission electron microscopy [8]. The conclusion that TEM underestimates cluster size concentration for small clusters is consistent with previous results by Katoh et al. [3], who reported TEM findings on neutron-irradiated $\mathrm{SiC}$ at $800^{\circ} \mathrm{C}$ up to $4.5 \times 10^{25} \mathrm{n} / \mathrm{m}^{2}$. In their study, the total estimated swelling from the loops was an order of magnitude smaller than swelling estimated from XRD. The discrepancy in our results between swelling values estimated from TEM and XRD is smaller than in the study of Katoh et al. [3]. This is due to the fact that we subtract the substrate strain from the total strain in the XRD analysis and account for contributions to swelling from isolated point defects, in addition to the 
contribution from clusters. We find that the contributions from all point defects constitute $38 \%$ of the total swelling calculated based on TEM and must therefore be included.

Another possible source of discrepancy between the XRD and TEM analyses of swelling is that the XRD data is averaged over a thicker specimen (bulk measurement) than typical TEM samples $(0.2 \mu \mathrm{m})$. The damage profile is depth-dependent, and corrections for such a damage distribution should be introduced to the TEM-based analysis in order to predict the overall swelling

of the sample. Such correction would require a detailed knowledge of the relation between local dpa and the defect size distribution. Additionally, XRD, as a bulk method, includes the swelling of the matrix caused by the implanted interstitials $(\mathrm{Kr}, \mathrm{C})$. For correction of the strain values from C-irradiation, concentration of the $\mathrm{C}$ antisite defects is needed. Assuming, all implanted $\mathrm{C}$ atoms rest in the interstitial positions in a $0.5 \mu \mathrm{m}$ region at the end of the irradiated zone (see Fig. 1), and taking the carbon interstitial formation volume from Tab. I and implantation fluence of $5.14 \times \times 10^{16} \mathrm{C} / \mathrm{cm}^{2}$, the strain values should be lower by 0.016 (about $3-5 \%$ ). If all implanted atoms end up as antisite defects then the strain values should be lowered by 0.029 (5-7.6\%). To correct the strain values for Kr-irradiation, information about formation volumes of $\mathrm{Kr}$ interstitials and $\mathrm{Kr}$ atoms in the vacancy position as well as concentration of $\mathrm{Kr}$ atoms in the vacancy position is required and it was not included in our modeling. However, since the Kr-irradiation fluence is two orders of magnitude lower than the $\mathrm{C}$ fluence, the effect of $\mathrm{Kr}$ interstitial should be negligible. The strain values obtained from TEM analysis are not disturbed by the presence of implanted interstitials, as the latter was performed at a depth were the damage zone was not disturbed by the implanted interstitials $(200 \mathrm{~nm}$ for $\mathrm{Kr}$ and $1500 \mathrm{~nm}$ for C. However, that means that swelling values measured by TEM should be used for swelling prediction for neutron-irradiated material, as neutron-irradiation does not introduce extraneous interstitials to the system which can contribute to swelling.

\section{SUMMARY}

The contribution of black spot defects and small clusters to the ion-induced swelling of singlecrystal 4H-SiC was studied by means of XRD and TEM combined with cluster dynamics simulations. The main results are as follows:

1. The damage zone underwent tensile strain. The total measured value of the strain is a 
result of the strain in the damage zone and the "substrate strain", which is the result of Poisson expansion. The tensile strain profile does not assume the shape of the damage profile calculated by SRIM and is rather quasi-flat throughout the entire damage zone, suggesting defect saturation, which is also confirmed by the lack of change in the swelling values with increasing damage level.

2. True strain, which is $84 \%$ of the total strain, reflects the actual swelling of the damage zone.

It was observed that as the irradiation temperature increased, the swelling decreased, which is attributed to defect recovery.

3. The swelling values obtained via TEM are usually lower by $40 \%$ to $70 \%$ than the XRD values, likely due to the lack of information about small clusters invisible to TEM. Point defects contribute $38 \%$ to the overall swelling, and the rest is due to the BSDs (interstitial clusters). Additionally, it is possible that XRD overestimates swelling, as it includes the swelling caused by a high fluence of implanted ions ( $\mathrm{C}$ and $\mathrm{Kr}$ ).

In general, the presented results improve our understanding of what defect types contribute to swelling, what is the magnitude of their contribution, and how their concentration depends on the damage level and the irradiation temperature.

\section{ACKNOWLEDGMENTS}

This research was funded by the DOE-NEUP grant \# 00128497. The authors would like to acknowledge Kim Kriewaldt, Benjamin Maier (UW-Madison), and Douglas Jeffers (UIUC) for their assistance during ion-irradiation. Instrumentation support was provided by NSF grant \# DMR-1121288.

[1] Y. Katoh, L. Snead, I. Szlufarska, and W. Weber, Current Opinion in Solid State and Materials Science 16, 143 (2012).

[2] L. Snead, T. Nozawa, Y. Katoh, T.-S. Byun, S. Kondo, and D. Petti, Journal of Nuclear Materials 371, 329 (2007).

[3] Y. Katoh, N. Hashimoto, S. Kondo, L. Snead, and A. Kohyama, Journal of Nuclear Materials 351, 228 (2006). 
[4] Y. Katoh, S. Kondo, and L. Snead, Journal of Nuclear Materials 382, 170 (2008).

[5] R. Devanathan and W. Weber, Journal of Nuclear Materials 278, 258 (2000).

[6] R. Stoller, M. Toloczko, G. Was, A. Certain, S. Dwaraknath, and F. Garner, Nuclear Instruments and Methods in Physics Research Section B: Beam Interactions with Materials and Atoms 310, 75 (2013).

[7] Y. Waseda, E. Matsubara, and K. Shinoda, X-ray diffraction crystallography (Springer, Berlin Heidelberg, 2011).

[8] C. Liu, H. Li, Y. Zhai, B. Tyburska-Püschel, P. Voyles, K. Sridharan, D. Morgan, and I. Szlufarska, Evolution of small defect clusters in ion-irradiated 3C-SiC: combined cluster dynamics modeling and experimental study (in preparation, 2016).

[9] J. Li, L. Porter, and S. Yip, Journal of Nuclear Materials 255, 139 (1998).

[10] J.-F. Barbot, M.-F. Beaufort, and A. Declémy, Nuclear Instruments and Methods in Physics Research Section B: Beam Interactions with Materials and Atoms 327, 59 (2014).

[11] C. Xu, C. Zhang, J. Li, L. Zhang, Y. Yang, Y. Song, X. Jia, J. Li, and K. Chen, Nuclear Instruments and Methods in Physics Research Section B: Beam Interactions with Materials and Atoms 286, 129 (2012).

[12] S. Leclerc, M. Beaufort, J. Barbot, and A. Declémy, Applied Physics Letters 93, 122101 (2008).

[13] J. Li, C. Zhang, C. Xu, X. Jia, Y. Song, and J. L. abd Y.F. Jin, Nuclear Instruments and Methods in Physics Research Section B: Beam Interactions with Materials and Atoms 286, 124 (2012).

[14] S. Leclerc, M. Beaufort, A. Declémy, and J. Barbot, Journal of Nuclear Materials 397, 132 (2010).

[15] H. Zang, D. Guo, T. Shen, C. He, Z. Wang, L. Pang, C. Yao, and T. Yang, Journal of Nuclear Materials 433, 378 (2013).

[16] J. Barbot, A. Declémy, and M. Beaufort, Physica Status Solidi A 210, 218 (2013).

[17] A. Debelle and A. Declémy, Nuclear Instruments and Methods in Physics Research Section B: Beam Interactions with Materials and Atoms 268, 1460 (2010).

[18] K. Kamitani, M. Grimsditch, J. Nipko, and C.-K. Loong, Journal of Applied Physics 82, 3152 (1997).

[19] A. Boulle and A. Debelle, Journal of Applied Crystallography 43, 1046 (2010).

[20] R. Price, Journal of Nuclear Materials 48, 47 (1973).

[21] D. Senor, G. Youngblood, L. Greenwood, D. Archer, D. Alexander, M. Chen, and G. Newsome, Journal of Nuclear Materials 317, 145 (2003).

[22] X. Wang, L. Jamison, K. Sridharan, D. Morgan, P. Voyles, and I. Szlufarska, Acta Materialia 99, 7 (2015). 
[23] S. Kondo, Y. Katoh, and L. Snead, Journal of Nuclear Materials 386-388, 222 (2009).

[24] Y.-R. Lin, C.-S. Ku, C.-Y. Ho, W.-T. Chuang, S. Kondo, and J.-J. Kai, Journal of Nuclear Materials 459, 276 (2015).

435

[26] http://www.srim.org/, (2015).

[27] T. Sawabe, M. Akiyoshi, K. Ichikawa, K. Yoshida, and T. Yano, Journal of Nuclear Materials 386-388, 333 (2009).

[28] G. Newsome, L. Snead, T. Hinoki, Y. Katoh, and D. Peters, Journal of Nuclear Materials 371, 76 440 (2007). 
TABLE I. Number of isolated point defects of a given type per cluster-trapped interstitials [8] and their formation volumes from Ref. [9].

\begin{tabular}{|c|c|c|c|c|c|c|}
\hline & $\mathrm{C}_{\mathrm{I}}$ & $\mathrm{Si}_{\mathrm{I}}$ & $\mathrm{V}_{\mathrm{C}}$ & $\mathrm{V}_{\mathrm{Si}}$ & $\mathrm{C}_{\mathrm{Si}}$ & $\mathrm{Si}_{\mathrm{C}}$ \\
\hline $\begin{array}{c}\text { Ratio of isolated point defects to } \\
\text { cluster-trapped interstitials } R\end{array}$ & $0.258 \times 10^{-8}$ & $0.110 \times 10^{-9}$ & 0.599 & 0.245 & 0.239 & 0.414 \\
\hline Formation volume $V_{\mathrm{IPD}}\left[\AA^{3}\right]$ & 15.33 & 37.22 & 2.68 & 1.85 & -9.52 & 15.44 \\
\hline
\end{tabular}


TABLE II. Summary of the swelling results from XRD and TEM for single-crystalline 4H-SiC irradiated by $3.15 \mathrm{MeV} \mathrm{C}$ up to $0.4 \mathrm{dpa}$ and $1 \mathrm{MeV} \mathrm{Kr}$ up to 0.4 and $0.8 \mathrm{dpa}$ at various temperatures. Swelling from XRD (at the nominal damage level) was calculated using the true strain values $\varepsilon_{\mathrm{N}}^{\mathrm{d}}$. Swelling from TEM was based on the BSD size and number density data shown in Fig. 7. For XRD swelling, the error bar of \pm 0.001 is defined by fitting a Gaussian curve into the satellite peak.

\begin{tabular}{|c|c|c|}
\hline Irradiation conditions & $\begin{array}{c}\text { Swelling XRD [\%] } \\
\text { (nominal dpa) }\end{array}$ & $\begin{array}{l}\text { Swelling TEM [\%] } \\
\text { (actual dpa) }\end{array}$ \\
\hline \multicolumn{3}{|l|}{ 3.15 MeV C: } \\
\hline $600^{\circ} \mathrm{C}$ & $0.580(0.4)$ & $0.623 \pm 0.094 \quad(0.37)$ \\
\hline $800^{\circ} \mathrm{C}$ & $0.466(0.4)$ & $0.228 \pm 0.042 \quad(0.34)$ \\
\hline $950^{\circ} \mathrm{C}$ & $0.381 \quad(0.4)$ & $0.223 \pm 0.032 \quad(0.32)$ \\
\hline \multicolumn{3}{|l|}{$1 \mathrm{MeV} \mathrm{Kr}:$} \\
\hline $600^{\circ} \mathrm{C}$ & $0.741 \quad(0.4)$ & $0.176 \pm 0.025 \quad(0.21)$ \\
\hline $600^{\circ} \mathrm{C}$ & $0.750(0.8)$ & $0.225 \pm 0.035 \quad(0.57)$ \\
\hline $800^{\circ} \mathrm{C}$ & $0.472(0.4)$ & $0.144 \pm 0.022 \quad(0.24)$ \\
\hline $800^{\circ} \mathrm{C}$ & $0.482(0.8)$ & $0.570 \pm 0.089 \quad(0.62)$ \\
\hline
\end{tabular}




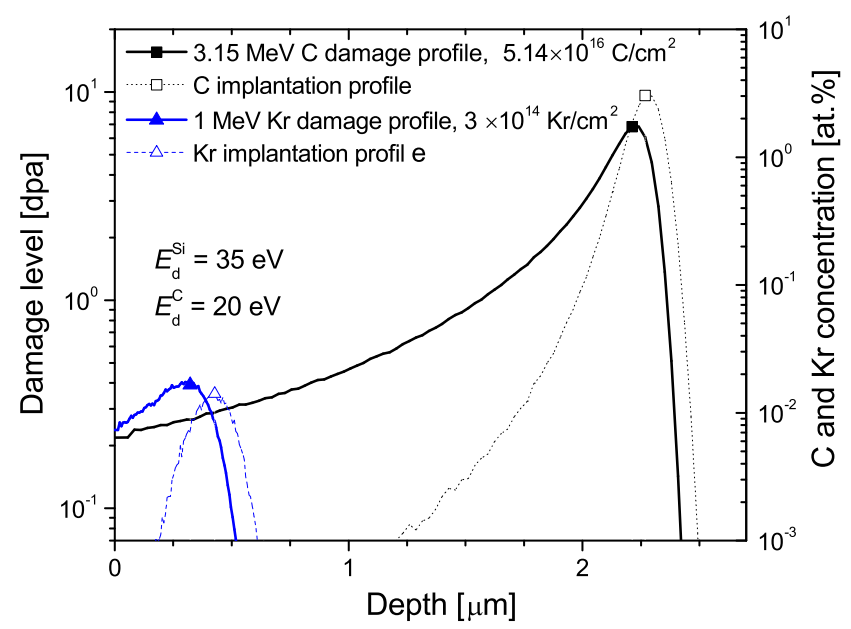

FIG. 1. Damage and $\mathrm{C}$ and $\mathrm{Kr}$ ion distributions in $\mathrm{SiC}$ irradiated with $3.15 \mathrm{MeV} \mathrm{C}$ and $1 \mathrm{MeV} \mathrm{Kr}$ ions to a damage level of $0.4 \mathrm{dpa}$ at the depth of $1 \mu \mathrm{m}$ and peak, respectively. Calculations were performed using SRIM-2013.00 [26], assuming the displacement threshold energies to be $20 \mathrm{eV}$ for $\mathrm{C}$ and $35 \mathrm{eV}$ for Si. 

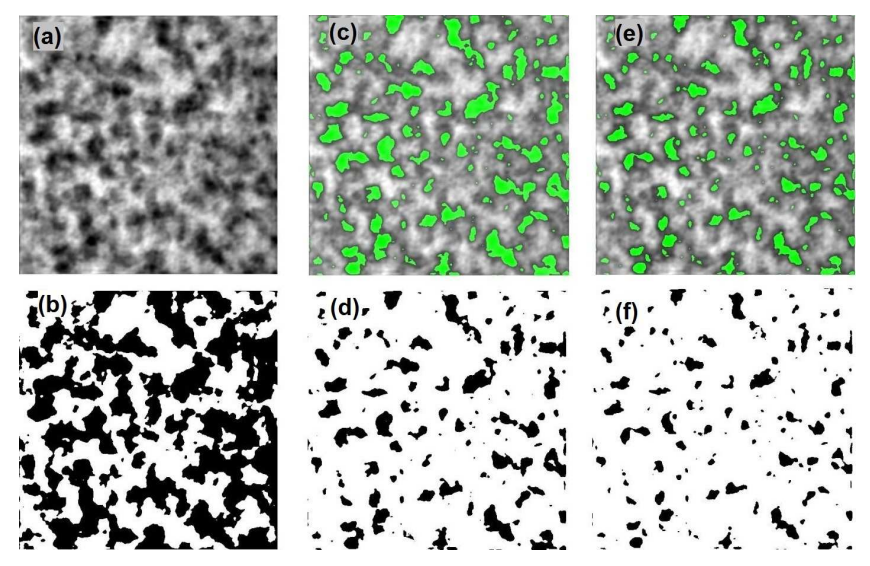

FIG. 2. (color online) Procedures of the local intensity method to identify BSDs: (a) Raw bright-field TEM image of irradiated 4H-SiC; (b) pattern of possible BSDs after $1^{\text {st }}$ threshold; (c) and (d) images processed after $2^{\text {nd }}$ threshold, black or dark mask shown as strain areas; (e) and (f) final images after $3^{\text {rd }}$ threshold. Grey patches (bright green online) represent regions below local threshold. 


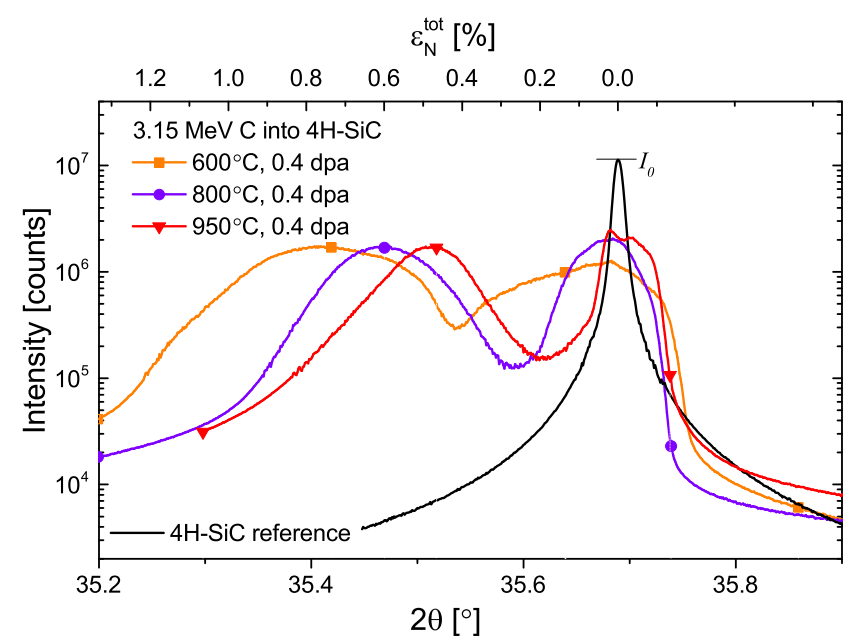

FIG. 3. (color online) $2 \theta-\omega$ scans at (0004) pole of $3.15 \mathrm{MeV}$ C-irradiated $4 \mathrm{H}-\mathrm{SiC}$ up to $0.4 \mathrm{dpa}$ at temperatures between $600^{\circ} \mathrm{C}$ and $950^{\circ} \mathrm{C}$. A $2 \theta-\omega$ scan from an un-implanted sample is shown for reference. With increasing temperature, the strain decreases. 

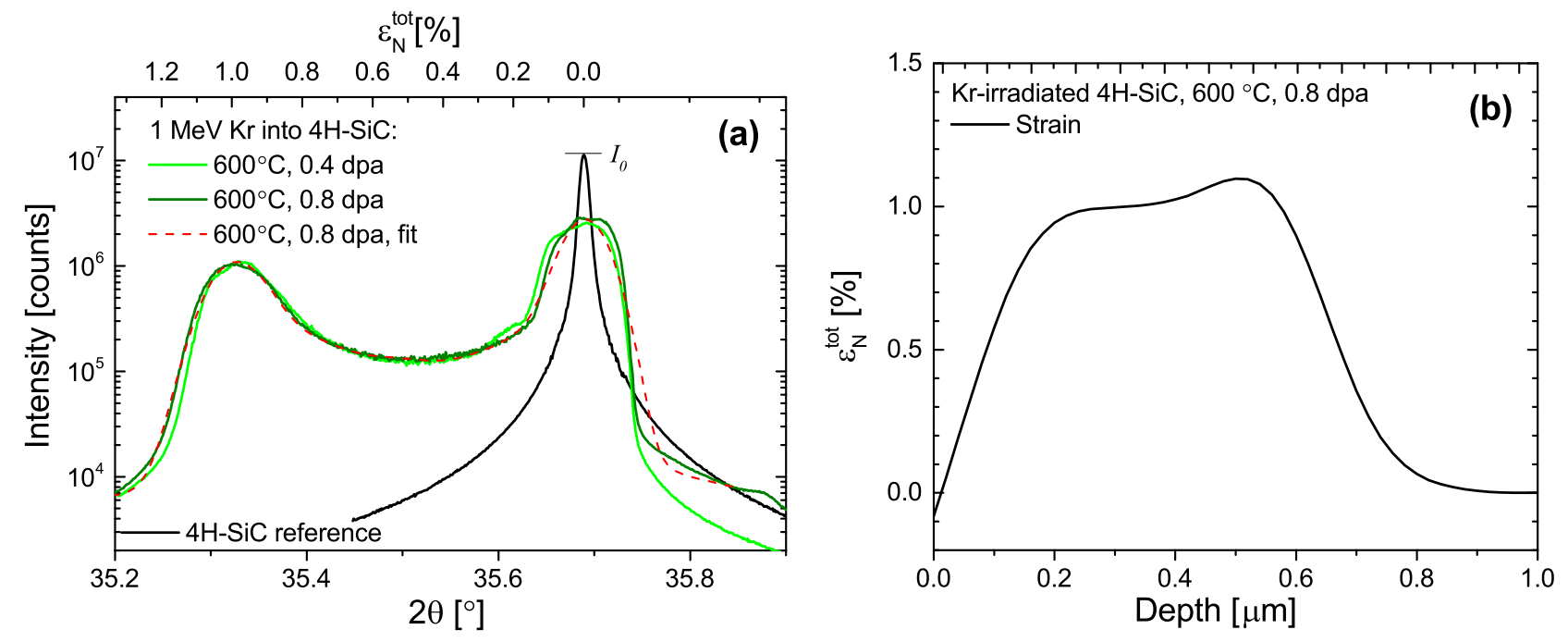

FIG. 4. (color online) (a) $2 \theta-\omega$ scans at the (0004) pole of $1 \mathrm{MeV} \mathrm{Kr}$-irradiated $4 \mathrm{H}-\mathrm{SiC}$ at $600{ }^{\circ} \mathrm{C}$ up to either 0.4 dpa or $0.8 \mathrm{dpa}$. A $2 \theta-\omega$ scan from an un-implanted sample is shown for reference. No difference in strain is observed above $0.4 \mathrm{dpa}$; (b) strain profile corresponding to $1 \mathrm{MeV} \mathrm{Kr}$-irradiated $4 \mathrm{H}-\mathrm{SiC}$ at $600{ }^{\circ} \mathrm{C}$ up to $0.8 \mathrm{dpa}$. 

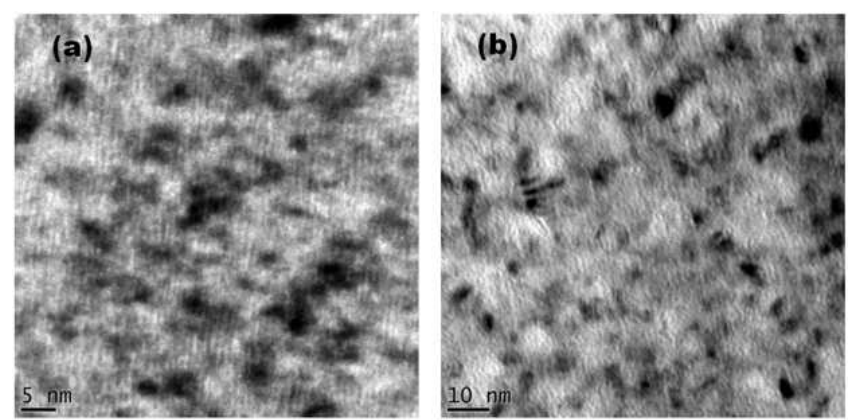

FIG. 5. Bright field TEM images of BSDs in (a) C-irradiated $4 \mathrm{H}-\mathrm{SiC}$ at $800^{\circ} \mathrm{C}$ and $0.51 \mathrm{dpa}$, and (b) Kr-irradiated $4 \mathrm{H}-\mathrm{SiC}$ at $800^{\circ} \mathrm{C}$ and 0.21 dpa. 


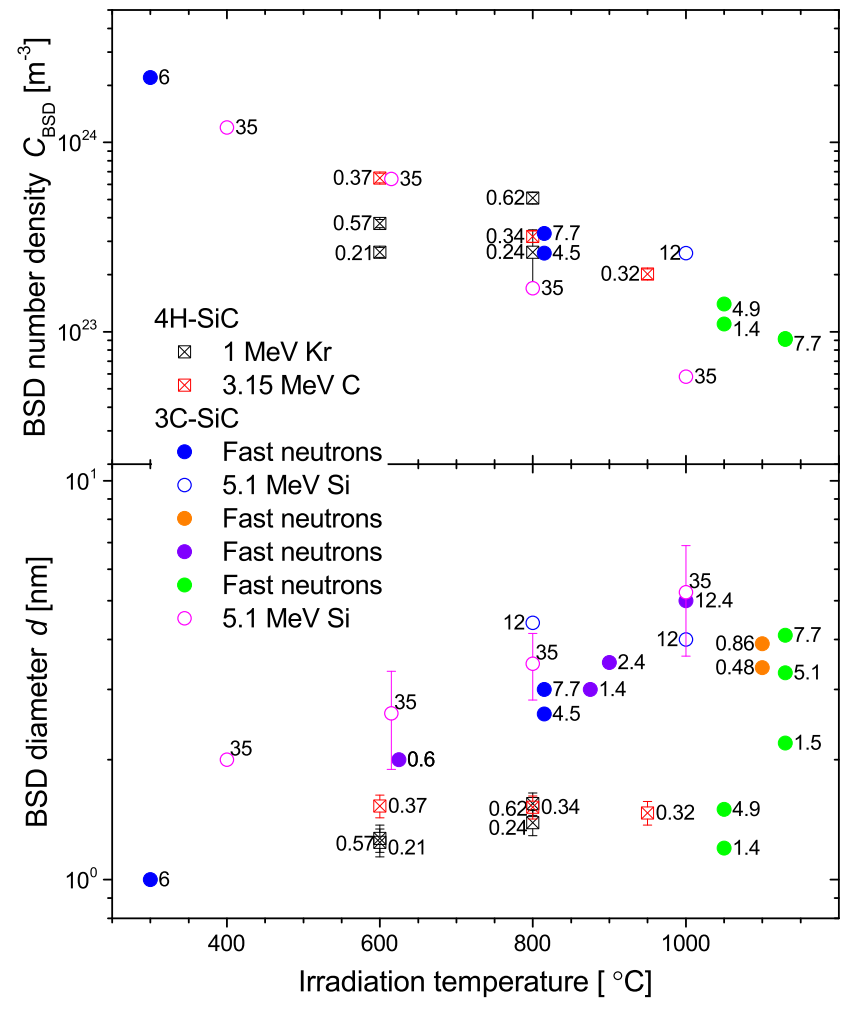

FIG. 6. (color online) Average BSD diameter and number density as a function of the irradiation temperature. The labels indicate the damage levels in dpa of exposed samples. With increasing temperature, BSDs coalescence such that their size increases and density decreases. Sources: $\nabla \nabla-$ this work, $\bullet \circ[3], \bullet[21]$, $\bullet[20], \bullet[23], \bigcirc[24]$. 


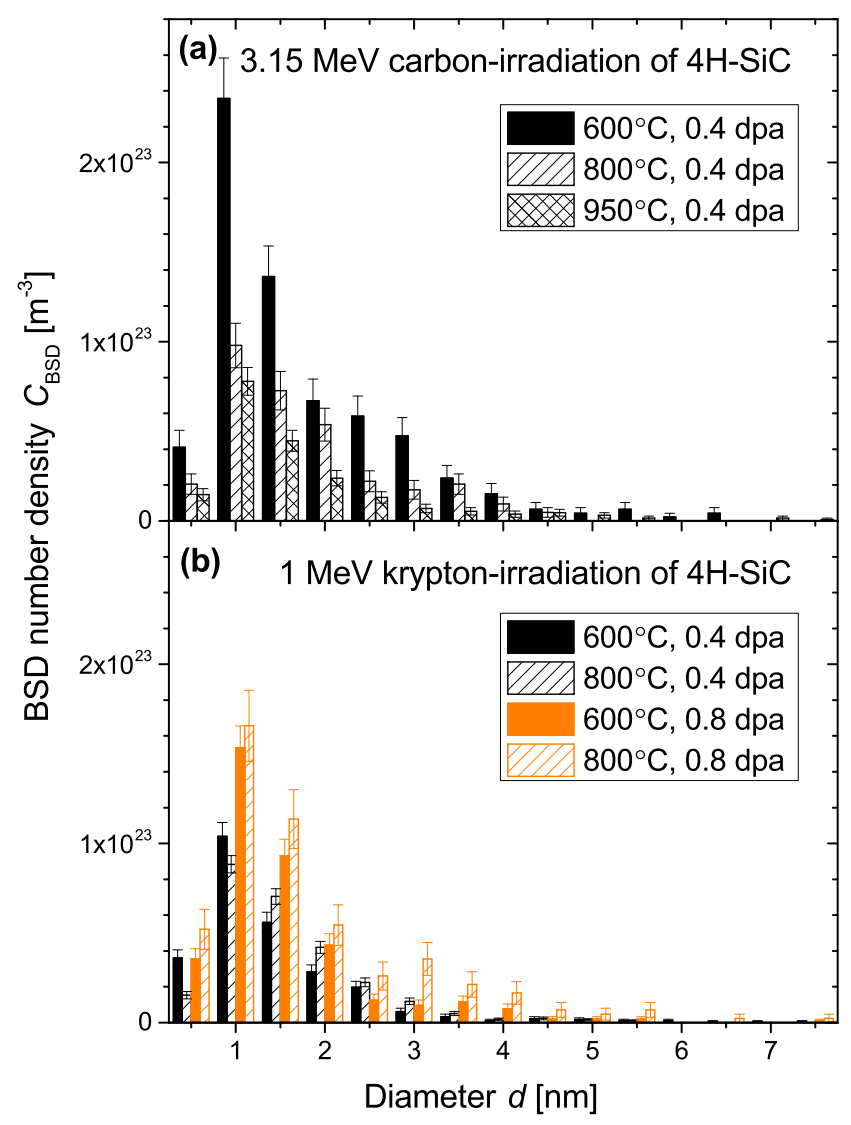

FIG. 7. (color online) BSD size distribution in single-crystalline $4 \mathrm{H}-\mathrm{SiC}$ irradiated by (a) $3.15 \mathrm{MeV} \mathrm{C}$ up to $0.4 \mathrm{dpa}$ and (b) $1 \mathrm{MeV} \mathrm{Kr}$ up to 0.4 and $0.8 \mathrm{dpa}$ at various temperatures. Here, the bin at label $d$ encompasses BSD sizes from $d-0.5$ to $d$. The actual damage level values in dpa are listed in Tab. II. 


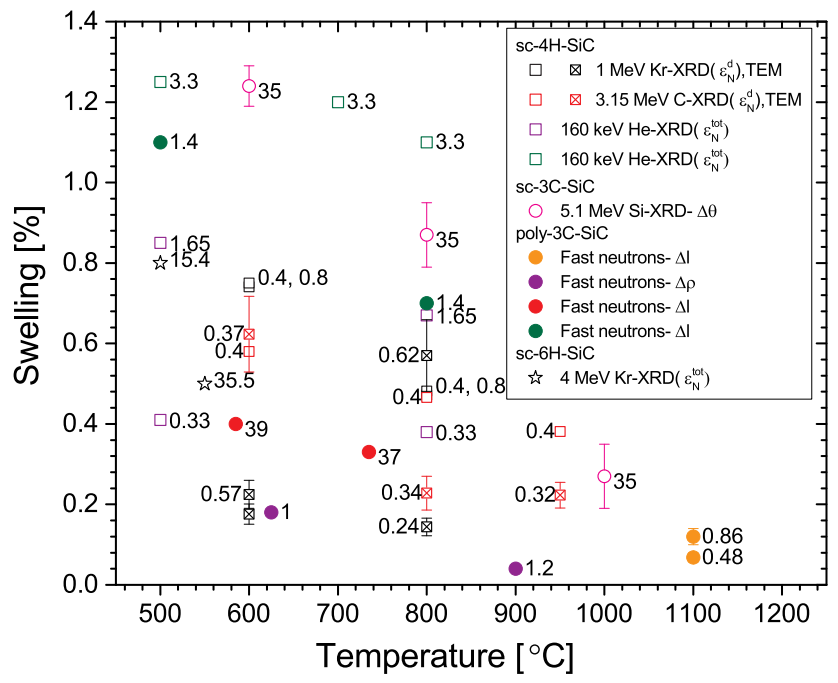

FIG. 8. (color online) Summary of swelling data measured by various methods $\left(\varepsilon_{\mathrm{N}}^{\mathrm{d}}\right.$ : true normal strain, $\varepsilon_{\mathrm{N}}^{\text {tot: }}$ total normal strain, $\Delta \theta$ : peak shift, $\Delta \rho$ : density change, $\Delta l$ : linear dimension change, and TEM) in neutron- (solid symbols) and ion-irradiated (empty symbols) 4H-SiC (squares), 3C-SiC (circles), and 6H$\mathrm{SiC}$ (pentagons). The labels indicate the damage levels in dpa of exposed samples. Sources: $\square \square \square \square-$ this work, $\square[14], \square[10,16], \bigcirc[24] \bullet[21], \bullet[20], \bullet[27], \bullet[28], \triangle[15]$. 